\title{
Assistência de enfermeiros da estratégia saúde da família a mulheres climatéricas com sintomas depressivos
}

\author{
Assistance from nurses in the family health strategy for climate women with depressive symptoms \\ Asistencia de enfermeras en la estrategia de salud familiar para mujeres climáticas con síntomas \\ depressivos
}

Recebido: 03/08/2021 | Revisado: 09/08/2021 | Aceito: 17/08/2021 | Publicado: 20/08/2021

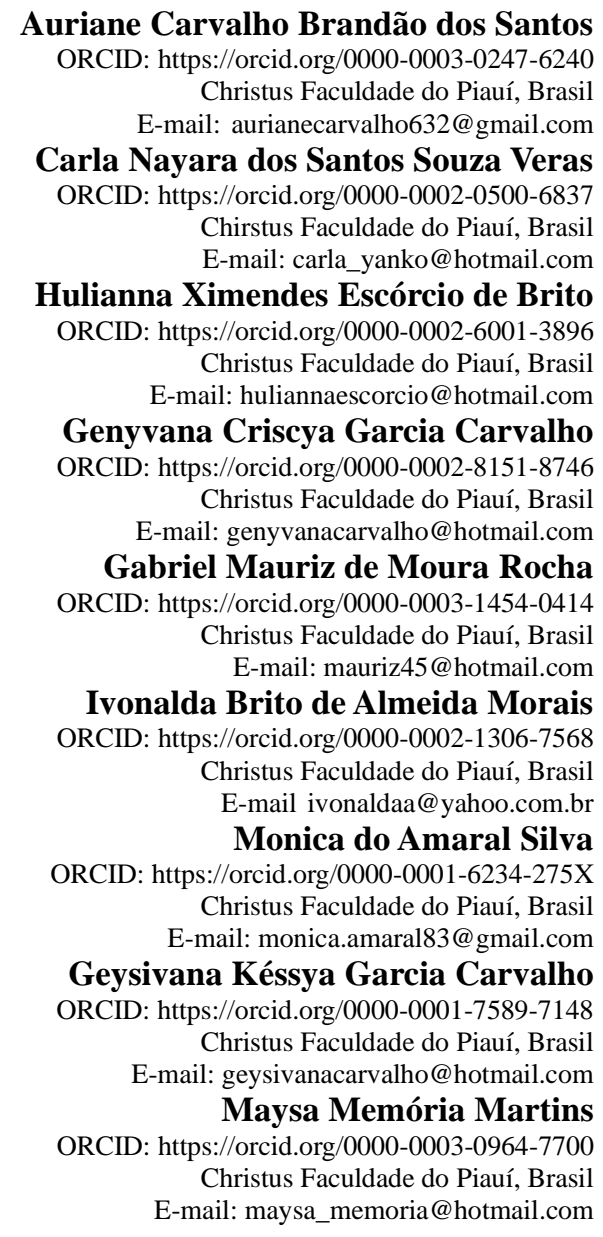

\section{Resumo}

As mulheres vêm exercendo diversos papéis na sociedade, ocupando espaços, que outrora, eram exclusivamente dos homens. $\mathrm{O}$ acúmulo de responsabilidades, atrelado às alterações hormonais que vivem durante as diferentes etapas da vida como a puberdade, puerpério e climatério culminam em maiores casos de depressão, quando comparado à população masculina. Objetivou-se com este estudo analisar a assistência de enfermeiros da ESF sobre sintomas depressivos em mulheres climatéricas, e como objetivos específicos relatar sobre o atendimento do enfermeiro às mulheres climatéricas com sintomas depressivos e mostrar a importância da assistência do enfermeiro na diferenciação da sintomatologia depressiva e climatérica. $\mathrm{O}$ estudo tratou de uma revisão bibliográfica integrativa da literatura. Após leitura minuciosa dos trabalhos encontrados de acordo com os critérios estabelecidos, permaneceram 7 artigos para discussão das categorias temáticas. Viu-se que a assistência é realizada por meio de atividades educacionais, os estudos com enfermeiros mostraram que os mesmos possuem dificuldade em prestar atendimento adequadamente, devido à falta de capacitações nesta área.

Palavras-chave: Depressão; Climatério; Cuidados de enfermagem.

\section{Abstract}

Women have been playing various roles in society, occupying spaces that were once exclusively for men. The accumulation of responsibilities, linked to hormonal changes that live during the different stages of life such as 
puberty, puerperium and climacteric culminate in greater cases of depression, when compared to the male population. The aim of this study was to analyze the assistance of FHS nurses on depressive symptoms in climacteric women, and as specific objectives to report on the care of nurses to climacteric women with depressive symptoms and to show the importance of nurse assistance in differentiating depressive symptoms and climacteric. The study dealt with an integrative literature review of the literature. After a thorough reading of the works found according to the established criteria, 7 articles remained for discussion of the thematic categories. It was seen that assistance is provided through educational activities, studies with nurses showed that they have difficulty in providing care properly, due to the lack of training in this area.

Keywords: Depression; Climacteric; Nursing care.

\section{Resumen}

Las mujeres han venido desempeñando diferentes roles en la sociedad, ocupando espacios que alguna vez pertenecieron a los hombres. La acumulación de responsabilidades, ligada a los cambios hormonales vividos durante las distintas etapas de la vida como la pubertad, el puerperio y el climaterio culminan en mayores casos de depresión, en comparación con la población masculina. El objetivo de este estudio fue analizar la atención de enfermeras de la ESF sobre síntomas depresivos en mujeres climatéricas, y como objetivos específicos informar sobre los cuidados brindados por enfermeras a mujeres climatéricas con síntomas depresivos y mostrar la importancia de los cuidados de enfermería en la diferenciación de los síntomas depresivos. y síntomas depresivos climatéricos. El estudio trató de una revisión bibliográfica integradora de la literatura. Luego de una lectura exhaustiva de los trabajos encontrados de acuerdo con los criterios establecidos, quedaron 7 artículos para discusión de las categorías temáticas. Se observó que la atención se brinda a través de actividades educativas, estudios con enfermeras han demostrado que tienen dificultad para brindar una atención adecuada, debido a la falta de capacitación en esta área.

Palabras clave: Depresión; Climatérico; Cuidado de enfermera.

\section{Introdução}

As mulheres vêm exercendo diversos papéis na sociedade, ocupando espaços, que, outrora, eram exclusivos dos homens. O acúmulo de responsabilidades, atrelado às alterações hormonais que ocorrem durante as diferentes etapas da vida como a puberdade, puerpério e climatério, culminam em maiores casos de depressão, quando comparado à população masculina (Coutinho et al., 2015).

De acordo com a Organização Mundial de Saúde (OMS), o climatério inicia a partir dos 35 anos e vai até os 65 anos, não sendo considerado um processo patológico, e sim um momento fisiológico e natural. A menopausa corresponde ao último ciclo menstrual, é vista como um marco na fase climatérica e reconhecida somente após doze meses da ocorrência da última menstruação (Lima et al., 2016; Silva, 2015).

Segundo Brasil (2016), as principais manifestações transitórias do climatério são: menstruais, tendo seu intervalo diminuído ou aumentado; neurogênicas, ondas de calor (fogachos), sudorese, calafrios, palpitações, cefaleia, tonturas, parestesias, insônia, perda de memória e fadiga; e psicogênicas, diminuição da autoestima, irritabilidade, labilidade afetiva, sintomas depressivos, dificuldades de concentração e dificuldades sexuais.

Em estudo desenvolvido com 48 mulheres no climatério, a maioria das participantes em relacionamento estável, pertencentes à classe média baixa, todas com remuneração, observou-se que 25\% (12/48) apresentaram manifestações depressivas com intensidade mínima a moderado. Esses episódios depressivos podem ser causados ou aumentados devido à presença de sintomas físicos (Real et al., 2018).

Em todo o mundo existem mais de 330 milhões de pessoas que sofrem de depressão, e somente $20 \%$ recebe tratamento adequado, quando diagnosticado corretamente. No Brasil, estima-se que 11,2 milhões apresentem sintomatologia depressiva. Dentre os vários sintomas incluem-se: energia diminuída, insônia ou hipersonia, agitação ou retardo, sentimentos de inutilidade, dificuldade de pensar ou pensamentos recorrentes de morte (Baptista, 2018; Santos, 2014).

Estatísticas mostram que o transtorno depressivo vem aumentando a cada ano e o risco é maior para mulheres, com índice que pode chegar a 18,6\%. Dados coletados em uma pesquisa realizada com usuários da atenção primária à saúde (APS), mostraram que a depressão apresenta-se como o segundo transtorno psiquiátrico mais prevalente nas mulheres, superado 
somente pelo transtorno de ansiedade generalizada (Machado, Oliveira e Delgado, 2013).

Sobretudo, a sintomatologia depressiva no climatério acontece com maior frequência na forma de distúrbios somáticos e psicológicos. Os somáticos caracterizam-se como síndromes de pernas inquietas, dores de cabeça, angina e distúrbios do trato intestinal. Já os sintomas psicológicos interferem na qualidade de vida da mulher climatérica, que favorece a suscetibilidade do surgimento da depressão. Com isso, evidencia-se que existe uma relação significativa entre a fase climatérica, presença de sintomas depressivos e alterações do humor (Santos, Pereira e Santos, 2016; Nogueira, 2018).

A Estratégia Saúde da Família (ESF) é considerada uma porta de entrada do Sistema Único de Saúde (SUS), integrada à saúde da família e permite aos profissionais desenvolverem uma prática assistencial dinâmica e integral. $\mathrm{O}$ enfermeiro, por ter função assistencialista, deve prestar atendimento à mulher no climatério considerando suas especificidades e aspectos psicobiológicos, o que exige capacitações deste profissional, para o desenvolvimento de estratégias de cuidados nesse período da vida da mulher (Oliveira e Wendhausen, 2014; Silva, 2015).

Tendo em vista o conjunto desses sintomas e sua semelhança, o enfermeiro pode encontrar dificuldade na diferenciação do que caracteriza os sintomas depressivos na fase climatérica. Diante deste contexto, questiona-se: Como é a assistência dos enfermeiros da Estratégia Saúde da Família em relação aos sintomas depressivos em mulheres climatéricas?

Para isso, diante a realização da pesquisa, apresentou-se como objetivo geral, analisar a assistência de enfermeiros da ESF sobre sintomas depressivos em mulheres climatéricas. E como objetivos específicos, relatar sobre o atendimento do enfermeiro às mulheres climatéricas com sintomas depressivos; mostrar a importância da assistência do enfermeiro na diferenciação da sintomatologia depressiva e climatérica.

\section{Metodologia}

A presente pesquisa tratou-se de uma revisão bibliográfica do tipo revisão integrativa, a qual se trata de um método que possui características marcadas pela inserção das evidências no exercício clínico, que tem como propósito agregar, mensurar e sintetizar resultados de trabalhos acerca de um determinado tema. Para a elaboração da pesquisa de revisão integrativa segue-se seis etapas: Identificação do tema e seleção da hipótese; estabelecer critérios para inclusão e exclusão de estudos; categorização dos estudos; avaliação dos estudos incluídos; interpretação dos resultados e síntese do conhecimento (Mendes, Silveira e Galvão, 2008).

A busca por artigos foi realizada no banco de dados Biblioteca Virtual em Saúde (BVS) e na base de dados Scientific Electronic Library Online (SciELO) e no buscador google scholar, por meio de Descritores em Ciências da Saúde (DeCS) controlados: depressão, climatério e cuidados de enfermagem. E os não controlados: sintomas depressivos, assistência de enfermagem, mulheres, menopausa, enfermagem e saúde da mulher. Realizou-se a busca integrando os descritores em pares e trios utilizando o operador booleano (AND).

Como critérios de inclusão, foram utilizados trabalhos publicados como artigo científico, disponíveis em texto completo, com livre acesso, que estivessem apropriados ao tema proposto e em línguas portuguesa e estrangeira.

Dentre os critérios de exclusão trabalhos que não estivessem conforme o tema proposto, trabalhos disponíveis em resumo ou incompleto e no caso de duplicidade dos estudos, nos respectivos bancos e bases de dados, preservou-se apenas o estudo de um deles, além de teses, dissertações, relatos, estudos de revisão integrativa e manuais.

Foram construídos dois instrumentos: os quadros sinópticos dos artigos selecionados. O primeiro quadro apresenta: títulos dos artigos, autores, ano, local/periódico, metodologia. E o segundo quadro contém os objetivos dos artigos encontrados e os principais achados.

Após a busca chegou-se a um total de 392 artigos a partir dos DECS controlados, que foram: depressão, climatério e cuidados de enfermagem e os descritores não controlados: sintomas depressivos, assistência de enfermagem, mulheres, 
menopausa, enfermagem e saúde da mulher. Após leitura minuciosa permaneceram somente 8 artigos que estavam dentre os critérios de inclusão.

\section{Resultados e Discussão}

Posteriormente a leitura dos artigos, os mesmos foram agrupados por similaridade e organizados em categorias de análise. Os trabalhos selecionados discorrem acerca da assistência dos enfermeiros da estratégia saúde da família a mulheres climatéricas com sintomas depressivos e totalizaram 7 estudos, estes organizados no Quadro 1:

Quadro 1 - Estudos selecionados.

\begin{tabular}{|c|c|c|c|c|c|}
\hline $\mathbf{N}^{\mathbf{o}}$ & TÍTULO & AUTORES & ANO & $\begin{array}{c}\text { LOCAL } \\
\text { E } \\
\text { PERIÓDICO }\end{array}$ & METODOLOGIA \\
\hline 1 & $\begin{array}{l}\text { Atenção ao climatério realizada por } \\
\text { profissionais da estratégia saúde da } \\
\text { família }\end{array}$ & $\begin{array}{l}\text { PEREIRA, A. B. } \\
\text { S et al. }\end{array}$ & 2016 & $\begin{array}{l}\text { Rio de Janeiro, Revista } \\
\text { de enfermagem UERJ }\end{array}$ & $\begin{array}{l}\text { Pesquisa descritiva, } \\
\text { transversal, exploratória }\end{array}$ \\
\hline 2 & $\begin{array}{l}\text { Intervención educativa de enfermería } \\
\text { para fomentar el autocuidado de la } \\
\text { mujer durante el climaterio }\end{array}$ & $\begin{array}{l}\text { MARTINEZ, M. } \\
\text { D et al. }\end{array}$ & 2016 & $\begin{array}{l}\text { México, Enfermagem } \\
\text { universitária }\end{array}$ & $\begin{array}{l}\text { Pesquisa qualitativa, } \\
\text { experimental e transversal }\end{array}$ \\
\hline 3 & $\begin{array}{l}\text { Epidemiologia da menopausa e dos } \\
\text { sintomas climatéricos em mulheres de } \\
\text { uma região metropolitana no sudeste } \\
\text { do Brasil: inquérito populacional } \\
\text { domiciliar }\end{array}$ & $\begin{array}{l}\text { FREDERICO } \\
\text { FILHO J. L. } \\
\text { et al. }\end{array}$ & 2015 & $\begin{array}{lr}\text { São Paulo, } & \text { Revista } \\
\text { Brasileira } & \text { de } \\
\text { Ginecologia } & \mathrm{e} \\
\text { Obstetrícia } & \end{array}$ & $\begin{array}{l}\text { Estudo exploratório e } \\
\text { transversal }\end{array}$ \\
\hline 4 & $\begin{array}{l}\text { Representações sociais elaboradas por } \\
\text { enfermeiras acerca da assistência à } \\
\text { mulher climatérica na atenção } \\
\text { primária }\end{array}$ & $\begin{array}{l}\text { SILVA, S.B; } \\
\text { NERY, I.S; } \\
\text { CARVALHO, } \\
\text { A.M.C }\end{array}$ & 2016 & $\begin{array}{l}\text { Teresina, Piauí Revista } \\
\text { Rene }\end{array}$ & Pesquisa qualitativa \\
\hline 5 & $\begin{array}{l}\text { Depressão e ansiedade em mulheres } \\
\text { climatéricas: fatores associados }\end{array}$ & $\begin{array}{l}\text { POLISSENI, A. F } \\
\text { et al. }\end{array}$ & 2009 & $\begin{array}{l}\text { Minas Gerais, Revista } \\
\text { Brasileira Ginecologia } \\
\text { obstétrica }\end{array}$ & $\begin{array}{l}\text { Estudo clínico, prospectivo e } \\
\text { transversal }\end{array}$ \\
\hline 6 & $\begin{array}{l}\text { Assistência à mulher no climatério: } \\
\text { discurso de enfermeiras }\end{array}$ & $\begin{array}{l}\text { LOPES, M. E. L } \\
\text { et. al }\end{array}$ & 2013 & $\begin{array}{l}\text { Recife, Revista de } \\
\text { enfermagem UFPE }\end{array}$ & $\begin{array}{l}\text { Estudo exploratório e } \\
\text { qualitativo }\end{array}$ \\
\hline 7 & $\begin{array}{l}\text { Depressão em mulheres climatéricas: } \\
\text { análise de mulheres atendidas } \\
\text { ambulatoriamente em um hospital } \\
\text { universitário no Maranhão }\end{array}$ & $\begin{array}{l}\text { SILVA, M. N. M } \\
\text { et.al }\end{array}$ & 2008 & $\begin{array}{l}\text { Rio Grande do Sul, } \\
\text { Revista de Psiquiatria }\end{array}$ & $\begin{array}{l}\text { Estudo prospectivo, analítico } \\
\text { e transversal }\end{array}$ \\
\hline 8 & $\begin{array}{l}\text { Prevalência de sintomas depressivos } \\
\text { no climatério }\end{array}$ & $\begin{array}{l}\text { ALCÂNTARA, } \\
\text { F.Z; ROSA, G. C. } \\
\text { L.; OREFICE, A. } \\
\text { F. L. }\end{array}$ & 2019 & $\begin{array}{l}\text { São Paulo, Unisanta } \\
\text { Health Science }\end{array}$ & Estudo transversal analítico. \\
\hline
\end{tabular}

Fonte: Autores (2020).

Os artigos selecionados encontraram-se com recorte temporal entre os anos 2008 a 2019. Os métodos utilizados pelos autores para construção dos estudos inclusos foram: um estudo de natureza descritiva, transversal e exploratória; um estudo clínico, prospectivo e transversal; dois estudos qualitativos e um estudo exploratório transversal, um estudo exploratório e 
qualitativo, um estudo transversal analítico e por fim, um estudo prospectivo, analítico e transversal. O Quadro 2 faz referência aos objetivos e os principais achados.

Quadro 2 - Objetivo e principais achados de estudos selecionados que respondem à pergunta de revisão.

\begin{tabular}{|c|c|c|}
\hline $\mathbf{N}^{o}$ & OBJETIVOS & PRINCIPAIS ACHADOS \\
\hline 1 & $\begin{array}{l}\text { Conhecer a assistência de enfermeiros } \\
\text { da ESF sobre sintomas depressivos em } \\
\text { mulheres climatéricas. }\end{array}$ & $\begin{array}{l}\text { Percebeu-se que a assistência é realizada através de apoio educacional que } \\
\text { consiste em ajudar o indivíduo, e como resultados mostrou que houve uma } \\
\text { mudança no padrão cognitivo/ perceptivo, e também uma melhora em } \\
\text { atividades como os cuidados dos sentidos e o exercício da mente. Este estudo } \\
\text { ainda nos mostra que após a intervenção de enfermagem com aplicação de } \\
\text { ações educativas, as mulheres demonstraram um interesse e vontade de } \\
\text { entender melhor esse estágio de sua vida. }\end{array}$ \\
\hline 2 & $\begin{array}{l}\text { Relatar sobre o atendimento do } \\
\text { enfermeiro às mulheres climatéricas } \\
\text { com sintomas depressivos. }\end{array}$ & $\begin{array}{l}\text { Nesta pesquisa os enfermeiros relataram dificuldades em atender mulheres } \\
\text { climatéricas, porém reconheceram a importância da assistência e expressaram } \\
\text { o desejo de participar de capacitação, a falta de conhecimento a respeito do } \\
\text { assunto pode ser a causa, de ainda não está instituída na rotina do trabalho. }\end{array}$ \\
\hline 3 & $\begin{array}{l}\text { Mostrar a importância da assistência do } \\
\text { enfermeiro na diferenciação da } \\
\text { sintomatologia depressiva e climatérica. }\end{array}$ & $\begin{array}{l}\text { Acredita-se que a prevalência elevada dessas alterações do humor, encontrada } \\
\text { nessa pesquisa, pode ser resultado de vários fatores atrelado à dificuldade que } \\
\text { essas mulheres têm de procurar atendimento psiquiátrico para transtornos } \\
\text { leves e moderados, devido ao estigma que a especialidade ainda carrega. }\end{array}$ \\
\hline 4 & $\begin{array}{l}\text { Obter uma compreensão mais } \\
\text { aprofundada sobrea qualificação de } \\
\text { profissionais da saúde na atenção } \\
\text { primária em relação ao climatério. }\end{array}$ & $\begin{array}{l}\text { Podemos notar através desse estudo, que a implantação da atenção à saúde da } \\
\text { mulher no climatério pressupõe a existência de profissionais de saúde } \\
\text { capacitados e sensibilizados para as particularidades inerentes a esse grupo } \\
\text { populacional vistas à melhoria da sua qualidade de vida. Geralmente, as } \\
\text { mulheres vivenciam essa etapa solitariamente, de forma silenciosa e com } \\
\text { informações insuficientes. Visto isso, considera-se a implementação de } \\
\text { intervenções no climatério, sendo valorizados, também os aspectos subjetivos } \\
\text { e culturais das queixas. }\end{array}$ \\
\hline 5 & $\begin{array}{l}\text { Verificar a presença de sintomas } \\
\text { depressivos em mulheres climatéricas. }\end{array}$ & $\begin{array}{l}\text { As mulheres têm um risco de } 2 \text { a } 4 \text { vezes maior, de apresentar um episódio } \\
\text { depressivo superior durante a perimenopausa e pós-menopausa precoce. Neste } \\
\text { estudo, observou-se associação entre maior intensidade de sintomas } \\
\text { climatéricos e apresentar depressão/ansiedade, e também associação com } \\
\text { autopercepção de estado de saúde ruim ou péssima. Essa relação pode ser } \\
\text { causada tanto pelo efeito prejudicial de um pior estado psicológico sobre os } \\
\text { sintomas da menopausa quanto pelo efeito negativo que os sintomas } \\
\text { climatéricos trazem ao humor e à autopercepção de saúde individual. }\end{array}$ \\
\hline 6 & $\begin{array}{l}\text { Analisar o conhecimento de } \\
\text { enfermeiras sobre o climatério }\end{array}$ & $\begin{array}{l}\text { Neste estudo, podemos ver a afirmação de algumas enfermeiras de não } \\
\text { possuir conhecimento adequado para atender a mulher no climatério, quando } \\
\text { estas buscam atendimento na Unidade de Saúde da Família. }\end{array}$ \\
\hline 7 & $\begin{array}{l}\text { Refletir sobre os fatores associados à } \\
\text { depressão no climatério. }\end{array}$ & $\begin{array}{l}\text { Sabe-se que essa fase da vida da mulher esta associada a um } \\
\text { hipoestrogenismo crescente, trazendo algumas mudanças clínicas e } \\
\text { psicológicas. Sendo assim, pode ser associado à depressão do humor e } \\
\text { influenciando na incidência de depressão. }\end{array}$ \\
\hline 8 & $\begin{array}{l}\text { Avaliar a prevalência de sintomas } \\
\text { depressivos em mulheres no climatério }\end{array}$ & $\begin{array}{l}\text { A diminuição de estrogênios circulantes na perimenopausa ocasiona sintomas } \\
\text { desconfortáveis que afetam o bem estar da mulher. Em decorrência do } \\
\text { hipoestrogenismo são observados: ondas de calor, enfraquecimento da } \\
\text { musculatura do assoalho pélvico, dispareunia, alterações de humor e sintomas } \\
\text { depressivos. Sabe-se que o hipoestrogenismo crescente acarreta em mudanças } \\
\text { clínicas e psicológicas, principalmente nos primeiros anos de climatério } \\
\text { podendo, portanto, acarretar na alteração do humor, propiciando o surgimento } \\
\text { de sintomas depressivos. }\end{array}$ \\
\hline
\end{tabular}


Buscou-se construir um diálogo entre os autores dos artigos selecionados por meio da discussão acerca da assistência de enfermeiros a mulheres com sintomas depressivos no climatério delimitando-se 02 categorias que melhor sistematizaram a temática: Caracterização da sintomatologia depressiva em mulheres climatéricas e A enfermagem e o cuidado às mulheres climatéricas com sintomas depressivos.

\subsection{Caracterização da sintomatologia depressiva em mulheres climatéricas}

De acordo com Pereira et al. (2016), o climatério é vivenciado sem necessidade do uso de medicamentos ou queixas por muitas mulheres, entretanto outras apresentam sintomas que variam em intensidade e diversidade, com isso, em ambos os casos, é essencial que haja um acompanhamento sistemático a essas mulheres.

Para Frederico Filho et al. (2015), o período climatérico pode afetar a qualidade de vida da mulher, pois se apresenta com sintomas variados, dentre eles destaca-se os vasomotores, como sudorese e fogachos, a atrofia genital e os transtornos psicológicos, acarretando em prejuízo pessoal e social.

De acordo com Silva et al. (2008), o surgimento dos episódios depressivos e a incidência de depressão pode estar associada ao hipoestrogenismo. Existe uma associação entre menopausa e a depressão, que podem ser desencadeadas em conjunto ou separadas aos fatores hormonais, socioculturais e comorbidades. Ainda no mesmo estudo, notou-se que os sintomas do humor parecem ter a menopausa como facilitadora e não como causadora.

Enquanto que, Polisseni et al. (2009), partindo de uma perspectiva psicossocial, argumentam que as mudanças no meio familiar, doença ou morte de familiares, diminuição de renda, divórcio e a síndrome do ninho vazio, são os causadores da depressão e ansiedade nas mulheres climatéricas e não são atribuídas as flutuações hormonais.

Menciona-se no estudo realizado por Silva et al. (2008), que a percepção da mulher sobre a menopausa representou uma variável associada com a depressão, do ponto de vista negativo. A ideia de que esta fase representa o ponto final na sua vida pessoal e profissional, é algo culturalmente enraizado e está ligado ao surgimento da depressão.

Compartilhando com esse pensamento, pesquisa realizada por Martinez et al. (2016) sobre a autopercepção/autoconceito, mostram que a posição assumida pelas mulheres no climatério, manifestada por sensação de perda, considerando a menstruação como um elemento decisivo em sua vida e quando desaparece trás consigo o sentimento de perda de si mesma, da sua própria identidade.

Segundo Polisseni et al. (2009), os dados de seu estudo, apresentaram como fator de risco para a síndrome depressiva, a presença de ansiedade, confirmando os dados evidenciados na literatura em outras pesquisas. Ressaltou também que nas três fases do climatério (pré- menopausa, menopausa e pós-menopausa), não houve diferença na ocorrência de depressão e ansiedade, apesar de ser observada uma prevalência elevada neste período de climatério. Destacando ainda que, um terço das mulheres sofrerá de depressão em sua vida, sendo que 9\% desses episódios predominam na fase do climatério.

O estudo de Alcântara, Rosa e Orefice (2019) demonstrou que a diminuição de estrogênios circulantes na fase perimenopausa ocasiona uma sintomatologia que afeta desconfortavelmente o bem estar da mulher. E, portanto, por essa diminuição hormonal observa-se queixas de ondas de calor, dor na relação sexual, alterações de humor e sintomas depressivos. Sabe-se que o hipoestrogenismo com o passar dos anos leva alterações clínicas e psicológicas, principalmente nos primeiros anos de climatério, podendo acarretar o humor, propiciando o surgimento de sintomas depressivos.

Diante disso, notou-se que ainda há controvérsias sobre a origem da associação de sintomas depressivos na fase do climatério, alguns autores defendem ser motivado por fatores hormonais, enquanto outros, acreditam ser por causas extrínsecas. Atualmente tem-se uma quantidade reduzida de estudos voltados a essa temática, contudo, isso trouxe reflexões de que a vida da mulher deve ser cuidada em todas estas etapas e não apenas em sua fase reprodutiva. 


\subsection{A enfermagem e o cuidado às mulheres climatéricas com sintomas depressivos}

Silva et al. (2008), em seu estudo evidenciam que é necessário a formação de profissionais de saúde capacitados para lidar com transtornos mentais, para que o atendimento seja adequado a uma parcela da população feminina, esta que apresenta crescimento contínuo.

Segundo Lopes et al. (2013), a educação em saúde é vista como um instrumento importante para a intervenção dos profissionais de saúde junto às mulheres no climatério, por meio das atividades educativas que podem colaborar na tentativa de esclarecer concepções equivocadas e preconceituosas sobre a fase do climatério, favorecendo o desenvolvimento de um novo olhar sobre essa fase.

Estudo realizado por Silva, Nery e Carvalho (2016), mostraram, segundo informações das enfermeiras, que para as mulheres se tornarem ativas no processo do climatério, é fundamental a realização de ações educativas. Nesta perspectiva, é notória a necessidade de maior condensação de esforço dos profissionais, especialmente de gestores da saúde e enfermeiros, em prepararem atendimento a esse público, com o objetivo de que as mulheres climatéricas tenham uma assistência mais qualificada, longevidade e mais qualidade de vida, de acordo com suas próprias possibilidades e mantenham suas funções mentais e físicas preservadas.

Martinez et al. (2016), ressaltam que para ocorrer intervenção em programas de saúde elaborados para mulheres climatéricas, faz-se necessário um amplo conhecimento sobre crenças, práticas e recursos populares que as mulheres usam para resolver seus problemas de saúde nesta fase, além de conhecimento científico em fisiopatologia, diagnóstico, sintomas, terapias e cuidados.

Os enfermeiros são os profissionais que mais participam das atividades de educação permanente propostas para a equipe da ESF, sendo este um local importante para oferecer assistência adequada à mulher na fase do climatério, pois atua, prioritariamente, na articulação entre promoção da saúde e a prevenção de agravos (PEREIRA et al., 2016).

Ao passo que, para Silva, Nery e Carvalho (2016), a assistência à mulher no climatério, é desorganizada, desarticulada e não inseridas no dia a dia, devido às ações ficarem na dependência de iniciativas individuais e da sensibilidade de cada profissional. Pôde-se perceber que as enfermeiras demonstraram que o atendimento à mulher climatérica na atenção primária, não atende à real necessidade da clientela.

Corroborando com este pensamento, Pereira et al. (2016), mostrou que a atenção à saúde da mulher, limita-se, frequentemente, ao período reprodutivo, prevenção de câncer de colo de útero e de mama e acompanhamento de doenças crônicas não transmissíveis. Foi identificada neste estudo, que a invisibilidade do climatério voltada às ações de saúde da mulher, pode coincidir com a mesma realidade de outras regiões ou outras unidades de saúde, pois há uma semelhança nos resultados de outras pesquisas nesta área e na ESF. Evidenciando assim, que as principais dificuldades no âmbito do atendimento à mulher climatérica são: a falta de visibilidade desta temática na formação e qualificação dos profissionais de saúde da ESF, bem como as condições inapropriadas de trabalho.

Visto que os estudos sobre a temática assistência de enfermagem em mulheres climatéricas e sintomas depressivos são escassos, no que se refere à assistência de enfermagem, pode-se notar que os enfermeiros tem dificuldades ou não possuem habilidades na diferenciação dos sintomas.

\section{Considerações Finais}

Os estudos mostraram que o climatério é uma fase em que a mulher vivencia mudanças em sua vida, e por vezes, essas mudanças podem trazer sentimentos diversos como: medo, incertezas, tristezas e outros, sendo ideal um acompanhamento profissional para identificar precocemente a presença de sintomas depressivos e assim realizar o atendimento adequado. 
Os objetivos do presente estudo foram alcançados, visto que, a assistência é realizada por meio de atividades educacionais, os estudos mostraram que os enfermeiros possuem dificuldades em prestar atendimento adequado, devido à falta de capacitações nesta área. Fazendo-se necessário que a implantação da atenção à saúde da mulher climatérica infere a existência de profissionais capacitados.

Portanto, é relevante ressaltar que este estudo revela a importância do enfermeiro, pois este se encontra-se à frente do cuidado, no que concerne à saúde com as mulheres, seus reforços notáveis, e junto à equipe multiprofissional, demonstram a importância das intervenções de enfermagem em todas as etapas da vida.

Contudo, houve alguns obstáculos durante a construção deste trabalho, como poucos estudos voltados ao tema, além da semelhança do conteúdo. Diante disso, faz-se necessário o desenvolvimento de pesquisas que contemplem a relação dessa temática: climatério e sintomas depressivos, uma vez que trazem inúmeras contribuições para a população. Bem como para os profissionais de saúde, visto que estarão cada vez mais habilitados para realizar as intervenções devidas, de acordo com a individualidade de cada mulher assistida.

\section{Referências}

Alcântara, F. Z., Rosa, G. C. L., \& Orefice, A. F. L. (2019). Prevalência de sintomas depressivos no climatério. Unisanta Health Science, 3 (1), $42-52$. https://periodicos.unisanta.br/index.php/hea/article/viewFile/1956/1498

Baptista, M. N (2018). Avaliando "depressões": dos critérios diagnósticos às escalas psicométricas. Avaliação Psicológica, 17(3), 301-310. http://pepsic.bvsalud.org/scielo.php?script=sci_arttext\&pid=S1677-04

Brasil, Ministério da Saúde (2016). Protocolos da Atenção Básica: saúde das mulheres. Brasília. http://bvsms.saude.gov.br/bvs/publicacoes/protocolos_atencao_basica_saude_mulheres.pdf

Coutinho, M. E. M. et al. (2015). Aspectos Biológicos e Psicossociais da Depressão Relacionado ao Gênero Feminino. Revista Brasileira de Neurologia e Psiquiatria, 19 (1). https://rbnp.emnuvens.com.br/rbnp/article/view/131

Frederico Filho. J. L. et al. (2015). Epidemiologia da menopausa e dos sintomas climatéricos em mulheres de uma região metropolitana no sudeste do Brasil: inquérito populacional domiciliar. Revista Brasileira Ginecologia Obstetrícia, 13083, 881. https://www.scielo.br/scielo.php?script=sci_arttext\&pid=S0100$72032015000400152 \&$ lang=pt

Lima, L. F. et al. (2016). Relação entre medidas antropométricas, escolaridade, renda e índice de qualidade da dieta de mulheres climatéricas. HU Revista, 42(4), 297-305. https://periodicos.ufjf.br/index.php/hurevista/article/view/2579:

Lopes, M. E. L et al. (2013). Assistência à mulher no climatério: discurso de enfermeiras. Revista de Enfermagem UFPE online, 7 (1), 665-71, 2013 https://pesquisa.bvsalud.org/brasil/resource/pt/bde-33285

Machado, R. M., Oliveira, S. A. B. M., \& Delgado, V. G. (2013). Características sociodemográficas e clínicas das internações psiquiátricas de mulheres com depressão. Revista Eletrônica de Enfermagem, 15 (1), 223-32. https://www.revistas.ufg.br/fen/article/view/14564

Martinez, M. D.et al. (2016). Intervenção educacional de enfermagem para incentivar autocuidado das mulheres durante o climatério. Enfermagem universitária. 13 (3), 142-150. https://sci-hub.tw/http://dx.doi.org/10.1016/j.reu.2016.04.001

Mendes, K S., Silveira, R. C. C. P., \& Galvão, C. M. (2008). Revisão integrativa, método de pesquisa para a incorporação de evidencias na saúde e na enfermagem. Texto Contexto enfermagem, 17 (4), 758-764, http://www.scielo.br/scielo.php?idosos e os diferentscript=sci_arttext\&pid=S010407072008000400018>

Nogueira, J. S. et al. (2018). Sintomas Psicológicos em Mulheres Climatéricas Cardiopatas. Cogitare Enfermagem, 23 (2). http://www.saude.ufpr.br/portal/revistacogitare/wpcontent/uploads/sites/28/2018/05/54075-233987-1-PB.pdf:

Oliveira, S. R. G., \& Wendhausen, Á. L. P. (2014). Significando a Educação em Saúde: Dificuldades e Possibilidades da Estratégia Saúde da Família. Trabalho, Educação e Saúde, 12 (1), 129-147. http://www.scielo.br/pdf/tes/v12n1/08.pdf

Pereira, A. B. S et al. (2016). Atenção ao climatério realizada por profissionais da estratégia saúde da família. Revista de enfermagem UFRJ, 24 (1), 13122. https://www.e-publicacoes.uerj.br/index.php/enfermagemuerj/article/view/13122/17861

Polisseni, A. F. et al. (2009). Depressão e ansiedade em mulheres climatéricas: fatores associados. Revista Brasileira de Ginecologia e Obstetrícia. 31 (1), 2834. https://www.scielo.br/scielo.php?script=sci_arttext\&pid=S0100-72032009000100006

Real, M. G. A. et al. (2018). Alterações biopsicossociais durante o climatério: sintomas somáticos, insônia e manifestações depressivas. Revista Kairós: Gerontologia, 21 (2), 09-30. http://ken.pucsp.br/kairos/article/view/40139

Santos, R. M. (2014). Associação entre depressão e a intensidade dos sintomas climatéricos na pós-menopausa. Rio de Janeiro. https://www.arca.fiocruz.br/handle/icict/10991 
Research, Society and Development, v. 10, n. 10, e577101019035, 2021

(CC BY 4.0) | ISSN 2525-3409 | DOI: http://dx.doi.org/10.33448/rsd-v10i10.19035

Santos, T. R., Pereira, S. V. M., \& Santos, R. L. (2016). Intensidade da sintomatologia climatérica em mulheres pós-menopausa. Revista da Rede de Enfermagem do Nordeste, 17 (2), 225-232. http://www.periodicos.ufc.br/rene/article/view/3005

Silva, C. B. et al. (2015). Atuação de Enfermeiros na atenção às Mulheres no Climatério. Revista de Enfermagem UFPE OnLine, $312-318$. https://periodicos.ufpe.br/revistas/revistaenfermagem/article/view/10341/11047

Silva, M. N. M. et al. (2008). Depressão em mulheres climatéricas: análise de mulheres atendidas ambulatorialmente em um hospital universitário no Maranhão. Revista de Psiquiatria do Rio Grande do Sul. 30 (2), 150-154. https://www.scielo.br/scielo.php?script=sci_abstract\&pid=S0101$81082008000300011 \& \operatorname{lng}=$ en\&nrm=iso\&tlng=pt

Silva, S. B., Nery, I. S., \& Carvalho, A. M. C. (2016). Representações sociais elaboradas por enfermeiras acerca da assistência à mulher climatérica na atenção primária. Revista Rene, 17 (3), 363-71. http://www.periodicos.ufc.br/index.php/rene/article/view/3469 